\title{
Adaptive Byte Compression and Decompression: A New Approach for Fractal Image Compression
}

\author{
Bhumika Gupta \\ Assistant Professor \\ Computer Science Engg. Deptt. \\ G.B.Pant Engg. College \\ Pauri Garhwal,Uttrakhand
}

\author{
Ashish Negi, Ph.D \\ Associate Professor \\ M.C.A. Deptt. \\ G.B.Pant Engg. College \\ Pauri Garhwal,Uttrakhand
}

\begin{abstract}
This paper addresses the area of image compression as it is applicable to various fields of image processing. On the basis of evaluating and analyzing the current image compression techniques this paper presents the Adaptive byte compression and decompression technique an approach applied to fractal image compression. It also includes various benefits of using image compression techniques
\end{abstract}

\section{Keywords}

Fractal,Self-similarity,IteratedFunction System(IFS) Fractal image compressin.

\section{INTRODUCTION TO IMAGE COMPRESSION}

Image compression can be defined as minimizing the size in bytes of a graphics file without degrading the quality of the image to an unacceptable level. This reduction allows more images to be stored in a given amount of memory space but the major benefit is the reduction of the time required for images to be sent over the Internet or downloaded from Web pages[1].

There are several different ways in which image files can be compressed like JPEG, GIF, PNG, fractals and wavelets.For Internet use, the two most common compressed graphic image formats are the JPEG format and the GIF format. The JPEG method is more often used for photographs, while the GIF method is commonly used for line art and other images in which geometric shapes are relatively simple[2]. Other method like fractals and wavelets offer higher compression ratios than the JPEG or GIF methods for some types of images. In near future PNG format will replace GIF format.

\section{FRACTAL IMAGE COMPRESSION}

The basic concept of fractal image compression is to use the characteristics of self similarity in an image Imagine a special type of photocopying machine that reduces the image to be copied by half and reproduces it three times on the copy (see fig.2.1). Now we will see that what is the result happens when we feed the output of this machine back as input. Fig. 2 shows several iterations of this process on several input images. We can observe that all the copies seem to converge to the same final image, the one in fig.2.2(c). Since the copying machine reduces the input image, any initial image placed on the copying machine will be reduced to a point as we repeatedly run the machine in fact, it is only the position and the orientation of the copies that determines what the final image looks like. The way the input image is transformed determines the final result when running the copy machine in a feedback loop. However we must constrain these transformations, with the limitation that the transformations must be contractive, that is, a given transformation applied to any two points in the input image must bring them closer in the copy, since if points in the copy were spread out the final image would have to be of infinite size.
Except for this condition the transformation can have any form [12].

In practice, choosing transformations of the form

$W_{i}\left[\begin{array}{l}x \\ y\end{array}\right]=\left[\begin{array}{l}a_{i} b_{i} \\ c_{i} d_{i}\end{array}\right]\left[\begin{array}{l}x \\ y\end{array}\right]+\left[\begin{array}{l}e_{i} \\ f_{i}\end{array}\right]$

is sufficient to generate interesting transformations called affine transformations of the plane. Each can skew, stretch, rotate, scale and translate an input image. A common feature of these transformations that run in a loop back mode is that for a given initial image each image is formed from a transformed (and reduced) copies of itself, and hence it must have detail at every scale. That is, the images are fractals[12].

Barnsley[12] suggested that perhaps storing images as collections of transformations could lead to image compression

This is the implication of fractal image compression. The extra detail needed for decoding at larger sizes is generated automatically by the encoding transforms. However in some cases the detail is realistic at low magnification and this is a useful feature of the method. Magnification of the original shows pixilation, the dots that make up the image are clearly discernable.

This is because of the magnification produced. Standard image compression methods can be evaluated using their compression ratios, the ratio of the memory required to store an image as a collection of pixels and the memory require to store a representation of the image in compressed form. The compression ratio of fractal is easy to misunderstand since the image can be decoded at any scale. In practice it is important to either give the initial and decompressed image sizes or use the same sizes for a proper evaluation, because the decoded image is not exactly the same as the original. Such schemes are said to be lossy.

In this paper the proposed work is extension of work conducted by Martin V Sewell [13], which is described in section 2.1.The main objective is to improve the compression ratio so that the performance of the overall system is increased.

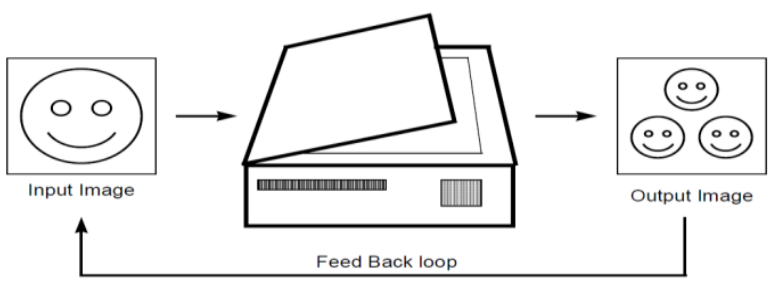

Figure 2.1: A copy machine that makes three reduced copies of the input image [Y] 
(a)
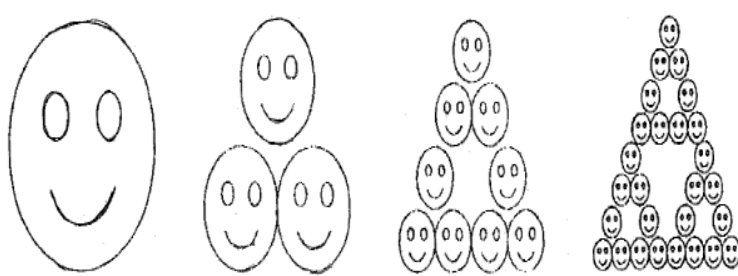

(b)

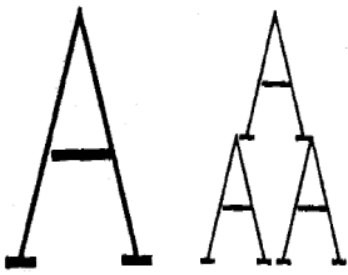

(c)
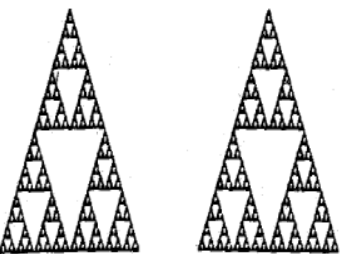

Initial Image First Copy
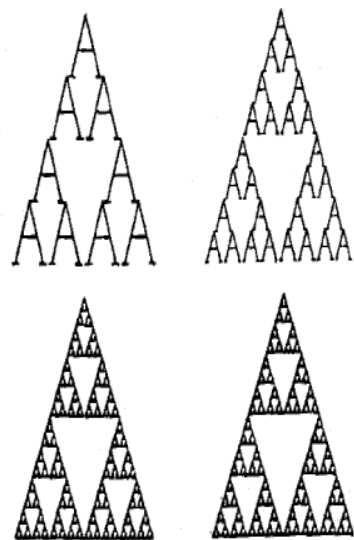

Second Copy

Figure 2.2: The first three copies generated on the copying machine Figure 2.1 [Y]

\subsection{The Fractal Compression Consists of Following Steps}

In Fractal image compression the encoding step is computationally expensive. A large number of sequential searches through a list of domains (portions of the image) are carried out while trying to find out the best match for another image partition Fractal image compression process starts with giving an image as input then in next step the image is split in $X$ $\mathrm{x} Y$ range and domain blocks after that we start with comparing the range with domain blocks then store similar blocks along with transformation applied after that similar blocks are constructed with pattern space hereby producing the fractal model from the pattern space and storing the result. The following steps for fractal image compression are show in fig.2.3.

\subsection{The New Approach Adaptive Byte Compression Algorithm}

Fractal image compression is time consuming in encoding process but compression ratio is also very important factor. In my work the major emphasis is on compression ratio, the proposed new approach i.e. Adaptive byte compression algorithm will increase the compression ratio to very high extend

The image format chosen to illustrate adaptive byte compression is that of .BMP and the program only supports black and white images The algorithm include Adaptive byte compression algorithm and Adative byte decompression algorithm.

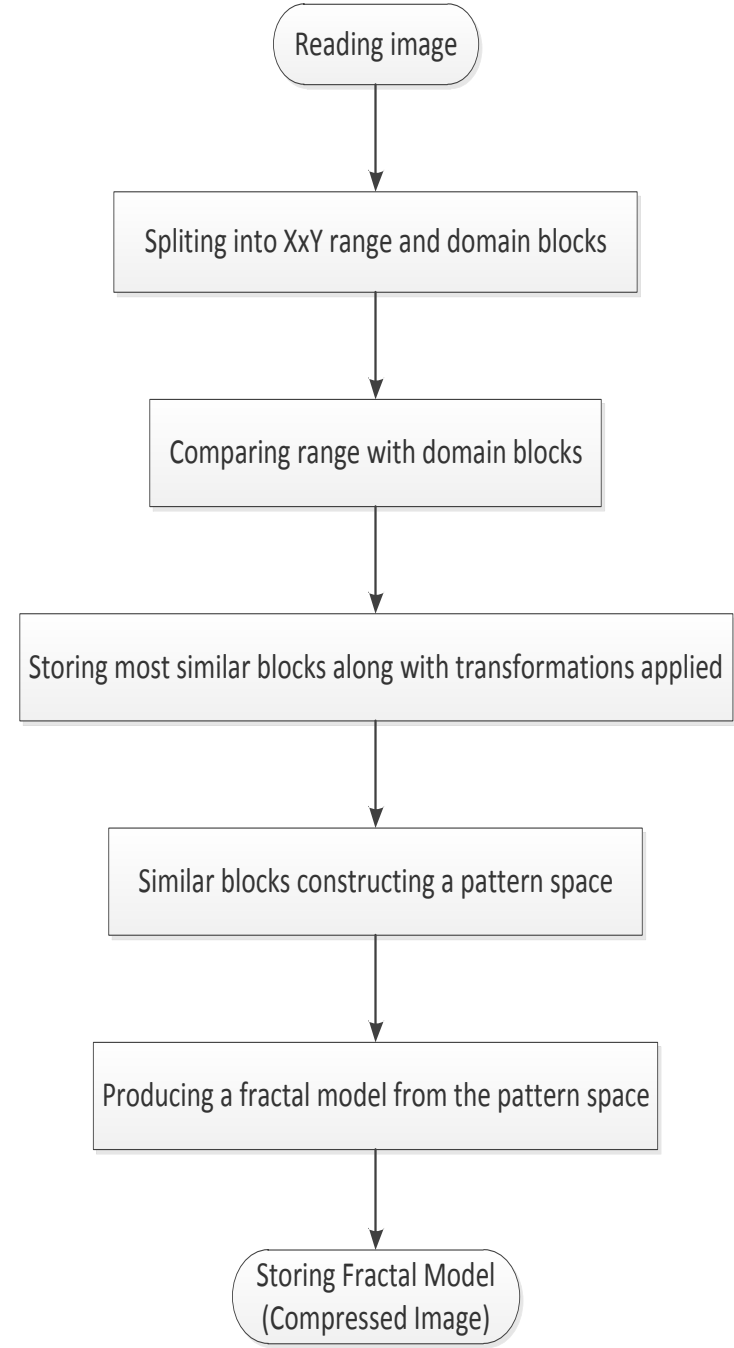

Figure 2.3: Steps of fractal image compression

\section{ADAPTIVE BYTE COMPRESSION ALGORITHM}

Adaptive byte compression algorithm is applied on the .tmp file which is the output of fractal image compression process as stated above in section 2.1. This is unique algorithm which analyze the input buffer (.tmp file) and parse it. The input buffer consists of patterns of bytes. The algorithm picks the first pattern (i.e. simplest pattern is 0x00) and counts the contiguous repetitions of pattern in the input buffer. It creates output buffer with the pattern at first location and followed by the count of pattern. This phenomenon is repeated throughout the input buffer taking care of buffer overflows and byte data limit overflows. Once completed for input buffer, the output buffer is created. The output buffer is called is .compressed file.

The steps of Adaptive byte compression is shown in fig.3.1. which also contains the steps of fractal image compression as input to the algorithm, so we can say that it is the extension of the fractal image compression to enhance the performance by increasing the compression ratio. 


\section{ADAPTIVE BYTE DECOMPRESSION ALGORITHM}

Adaptive byte decompression algorithm is applied on the .compressed file which is the final output of adaptive byte compression algorithm. This is unique algorithm which analyze the output buffer (.compressed file) and parse it. The output buffer consists of pattern and pattern repetition count. The algorithm picks the first pattern (i.e. simplest pattern is 0x00) and the count (followed by the pattern). It creates input buffer with the pattern at first location and the contiguous repetitions of pattern for the count specified. This phenomenon is repeated throughout the output buffer. Once completed for output buffer, the input buffer is created. The input buffer is called is .tmp file. The .tmp file is input for fractal decompression algorithm. The new approach applied to fractal compression algorith i.e adaptive byte compression algorithm showed in fig.3.1. consists of following steps-

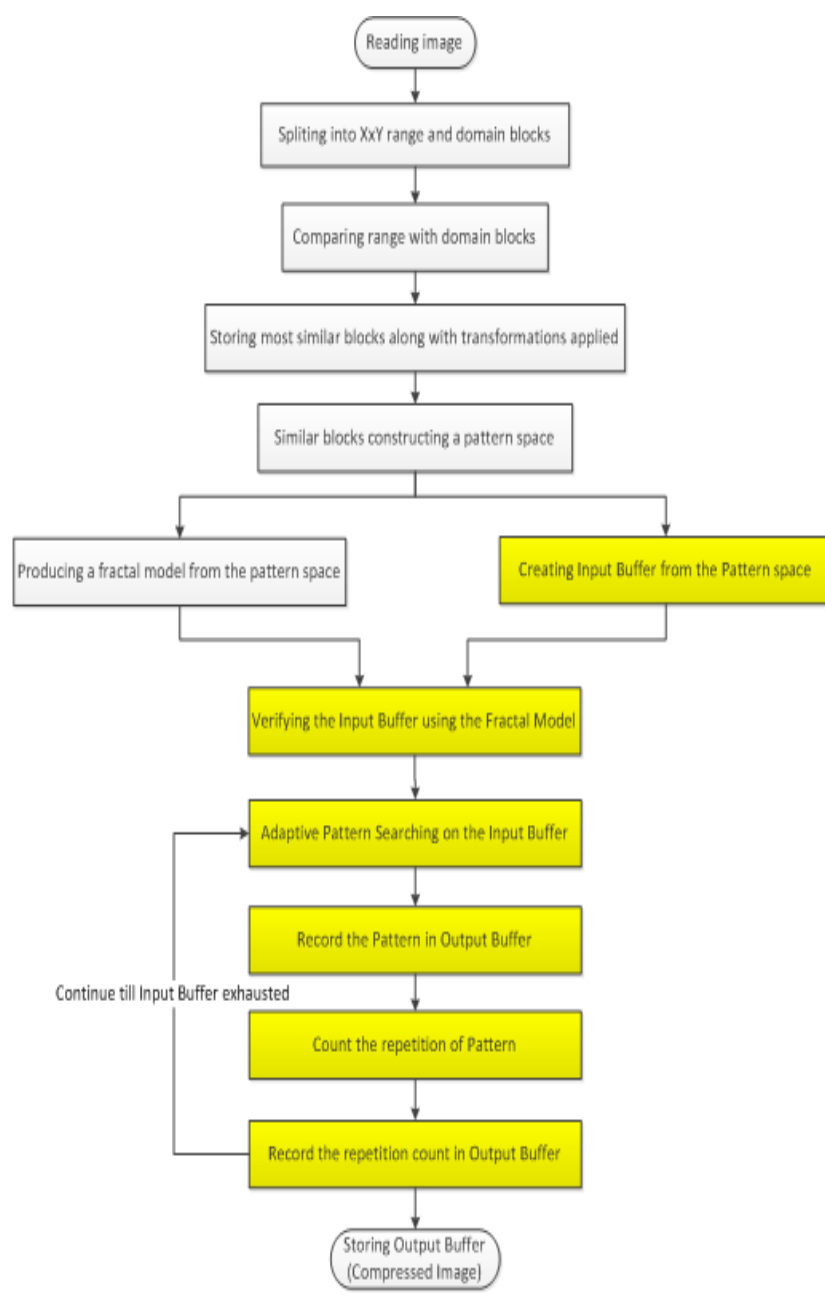

Figure.3.1. Steps of Adaptive byte compression algorithm

\section{COMPRESSION RATIO}

The objective of image compression is to reduce irrelevance and redundancy of the image data in order to be able to store or transmit data in an efficient form. Compression ratio can be defined as the size of the compressed file compared to that of the uncompressed file.

Fractal Compression \% and Ratio is calculated by comparing the compressed image size with the original image size. The compression ratio provides the efficiency of compression algorithm.
Fractal compression $\%=\frac{\text { compressed } \text { image size }}{\text { original } \text { image size }} \times 100$

Fractal compression ratio $=\frac{\text { original image size }}{\text { compressed image size }}: 1$

Adaptive Fractal Compression \% and Ratio is calculated by comparing the adaptive byte compressed image size with the original image size. The adaptive compression ratio provides the efficiency of adaptive byte compression algorithm.

$$
\begin{aligned}
& \text { Adaptive Fractal compression } \%=100-\frac{\text { Adaptive byte compressed image size }}{\text { original image size }} \times 100 \\
& \text { Adaptive Fractal compression ratio }=\frac{\text { original image size }}{\text { Adaptive byte compressed image size }}: 1
\end{aligned}
$$

\section{TABLE OF RESULTS}

Table 6.1. Size of original image

\begin{tabular}{|c|c|c|}
\hline Original Image & $\begin{array}{l}\text { Size of } \\
\text { Image/pixel } \\
\text { (width x Height) }\end{array}$ & $\begin{array}{c}\text { Size of file in } \\
\text { bytes }\end{array}$ \\
\hline Signature1.bmp & $512 \times 512$ & 32830 bytes \\
\hline Signature2.bmp & $128 \times 384$ & 6206 bytes \\
\hline Signature3.bmp & $256 \times 512$ & 16446 bytes \\
\hline Signature4.bmp & $512 \times 512$ & 32830 bytes \\
\hline Signature5.bmp & $254 \times 512$ & 16446 bytes \\
\hline Signature6.bmp & $510 \times 512$ & 32830 bytes \\
\hline Portrait.bmp & $550 \times 382$ & $27566 b y t e s$ \\
\hline horse.bmp & $568 \times 243$ & 17558 bytes \\
\hline breeze.bmp & $534 \times 357$ & 24338 bytes \\
\hline
\end{tabular}

Table 6.2. Size of fractal compressed file

\begin{tabular}{|c|c|}
\hline Original Image & Size of .tmp file in bytes \\
\hline Signature1.bmp & 25194 bytes \\
\hline Signature2.bmp & 6050 bytes \\
\hline Signature3.bmp & 14086 bytes \\
\hline Signature4.bmp & 25194 bytes \\
\hline Signature5.bmp & 11170 bytes \\
\hline Signature6.bmp & 31022 bytes \\
\hline Portrait.bmp & 17466 bytes \\
\hline horse.bmp & 12870 bytes \\
\hline breeze.bmp & 16782 bytes \\
\hline
\end{tabular}


Table 6.3. Size of adaptive fractal compressed file

\begin{tabular}{|c|c|}
\hline Original Image & $\begin{array}{c}\text { Size of .compressed file in } \\
\text { bytes }\end{array}$ \\
\hline Signature1.bmp & 4422 bytes \\
\hline Signature2.bmp & 1494 bytes \\
\hline Signature3.bmp & 2956 bytes \\
\hline Signature4.bmp & 4420 bytes \\
\hline Signature5.bmp & 1790 bytes \\
\hline Signature6.bmp & 15580 bytes \\
\hline Portrait.bmp & 7366 bytes \\
\hline horse.bmp & 8378 bytes \\
\hline breeze.bmp & 7698 bytes \\
\hline
\end{tabular}

Table 6.4. Fractal compression percentage and Fractal compression ratio

\begin{tabular}{|c|c|c|}
\hline $\begin{array}{c}\text { Original } \\
\text { Image }\end{array}$ & $\begin{array}{c}\text { Compression \% } \\
\text { (Fractal } \\
\text { compression \%) }\end{array}$ & $\begin{array}{c}\text { Compression } \\
\text { ratio } \\
\text { (Fractal } \\
\text { compression } \\
\text { ratio) }\end{array}$ \\
\hline Signature1.bmp & 23.36 & $1.30: 1$ \\
\hline Signature2.bmp & 02.51 & $1.03: 1$ \\
\hline Signature3.bmp & 14.35 & $1.17: 1$ \\
\hline Signature4.bmp & 23.26 & $1.30: 1$ \\
\hline Signature5.bmp & 32.08 & $1.47: 1$ \\
\hline Signature6.bmp & 05.51 & $1.06: 1$ \\
\hline Portrait.bmp & 36.64 & $1.58: 1$ \\
\hline horse.bmp & 26.70 & $1.36: 1$ \\
\hline breeze.bmp & 31.05 & \\
\hline & & \\
\hline & & \\
\hline & & \\
\hline & & \\
\hline & & \\
\hline & & \\
\hline & & \\
\hline & & \\
\hline
\end{tabular}

Table 6.5. Adaptive Fractal compression percentage and ratio

\begin{tabular}{|c|c|c|}
\hline Original Image & $\begin{array}{c}\text { Compression } \\
\% \\
\text { (Adaptive } \\
\text { Fractal } \\
\text { compression } \\
\% \text { ) }\end{array}$ & $\begin{array}{c}\text { Compression } \\
\text { ratio } \\
\text { (Adaptive } \\
\text { Fractal } \\
\text { compression } \\
\text { ratio) }\end{array}$ \\
\hline Signature1.bmp & 86.53 & $7.42: 1$ \\
\hline Signature2.bmp & 75.93 & $4.15: 1$ \\
\hline Signature3.bmp & 82.03 & $5.56: 1$ \\
\hline Signature4.bmp & 86.54 & $7.43: 1$ \\
\hline Signature5.bmp & 89.12 & $9.19: 1$ \\
\hline Signature6.bmp & 52.54 & $2.11: 1$ \\
\hline Portrait.bmp & 73.28 & $3.74: 1$ \\
\hline horse.bmp & 52.28 & $2.10: 1$ \\
\hline breeze.bmp & 68.37 & $3.16: 1$ \\
\hline
\end{tabular}

\section{IMAGES (ALL SAMPLES ARE EXECUTED OUTPUTS OF THE PROGRAM)}

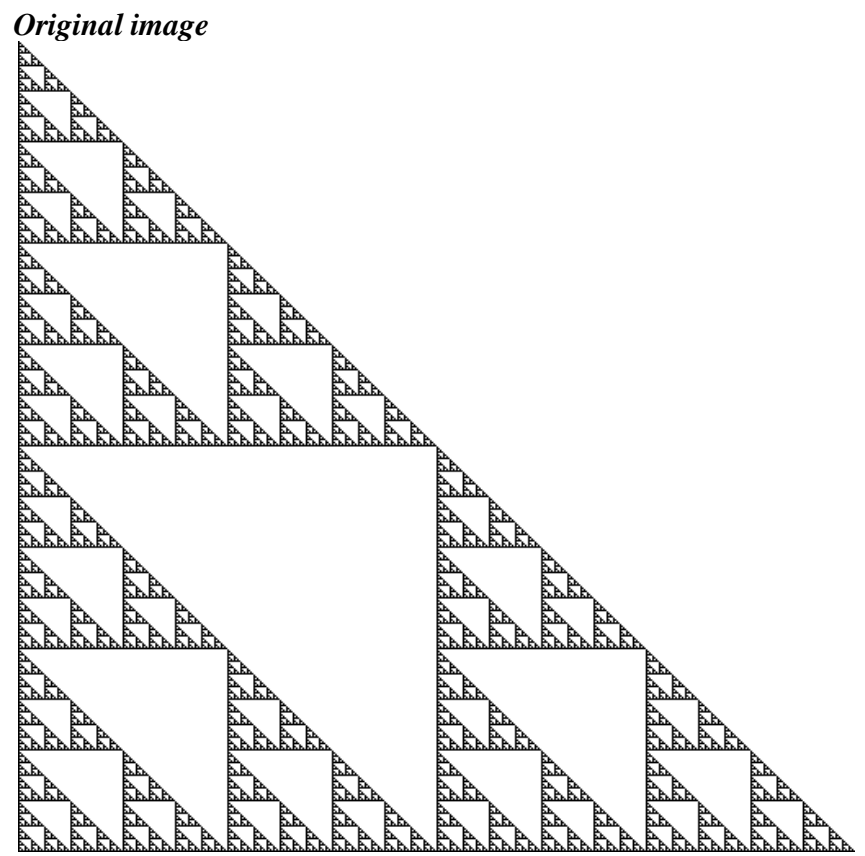

Figure 1. Signature1 


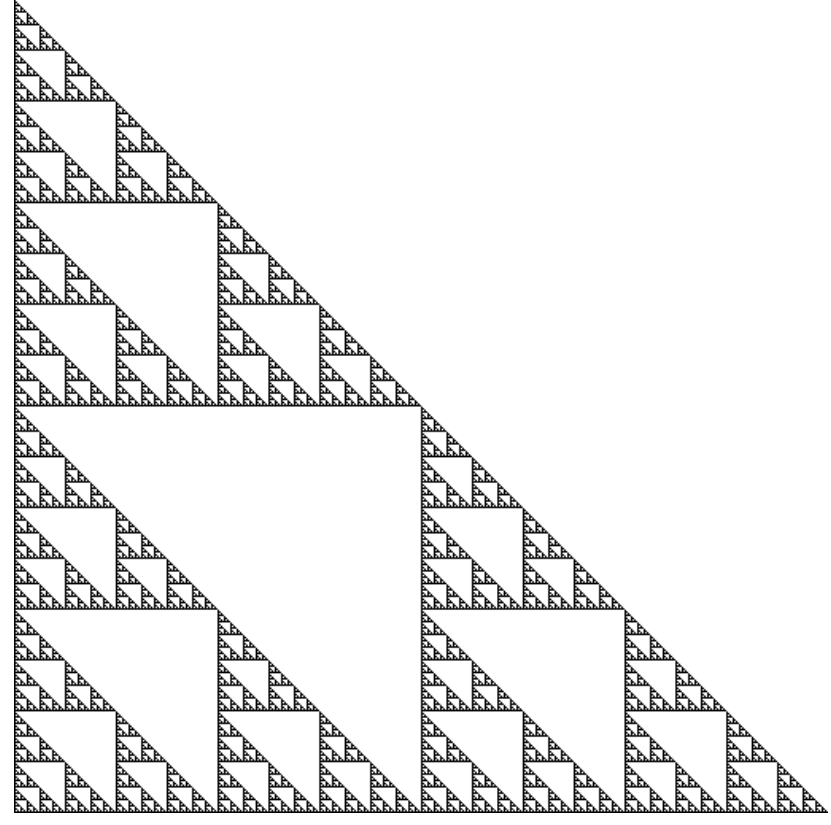

De-compressed Image (Adaptive Byte Fractal Decompression Output)

\section{Original Image}

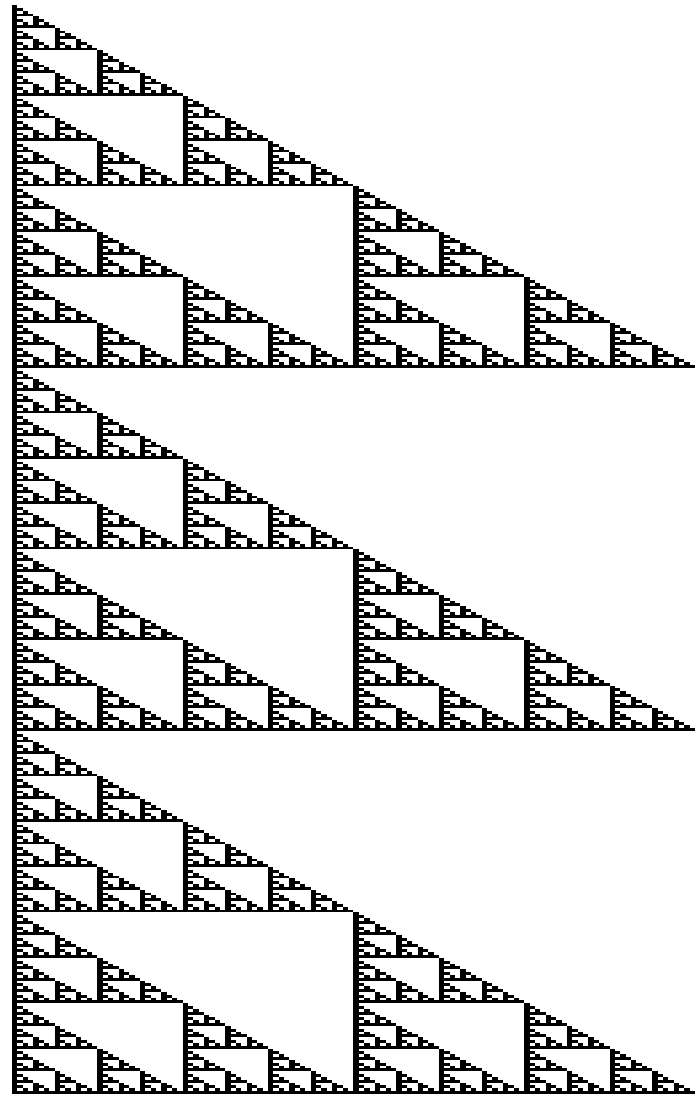

Figure 2. Signature2

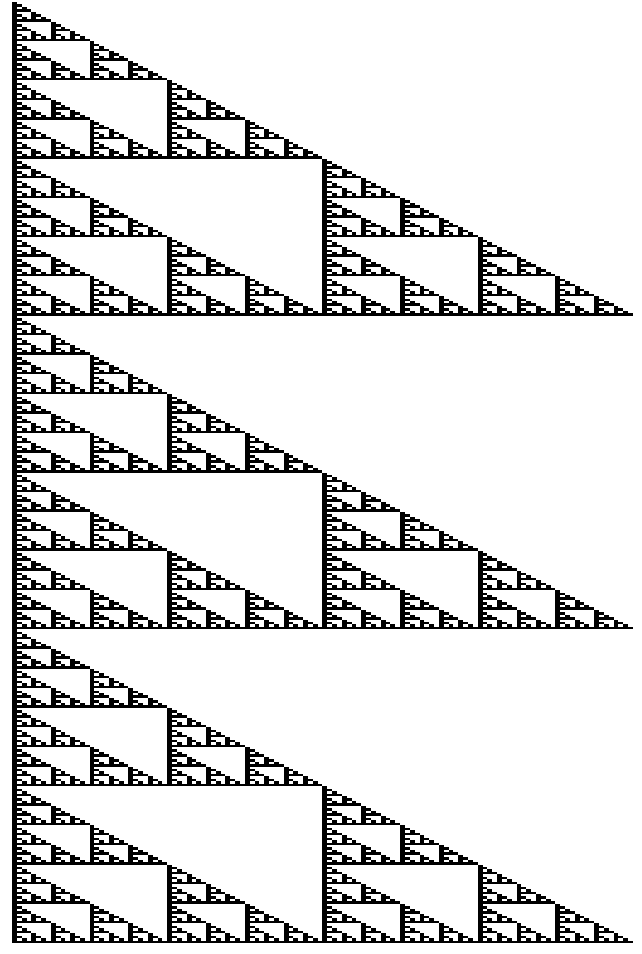

De-compressed Image (Adaptive Byte Fractal Decompression Output)

\section{Original Image}

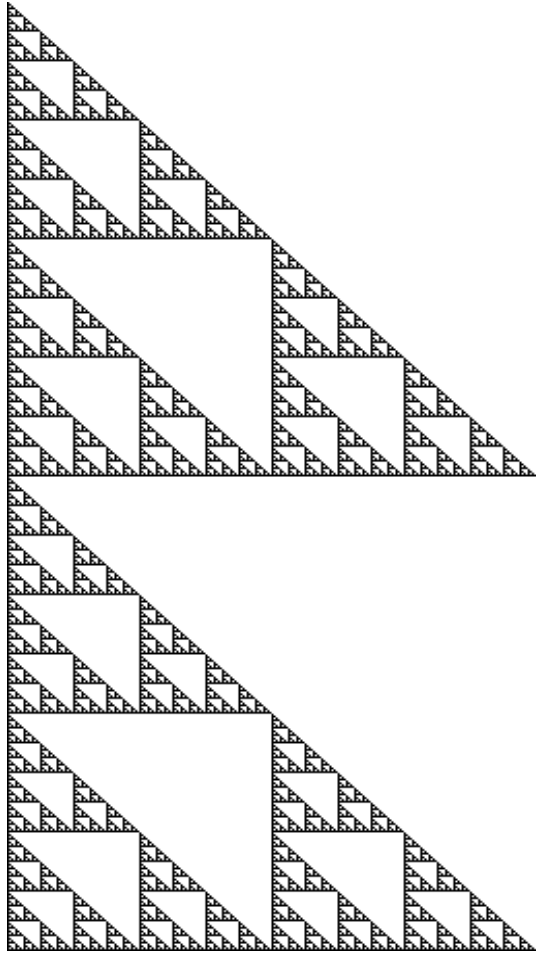

Figure 3. signature3 


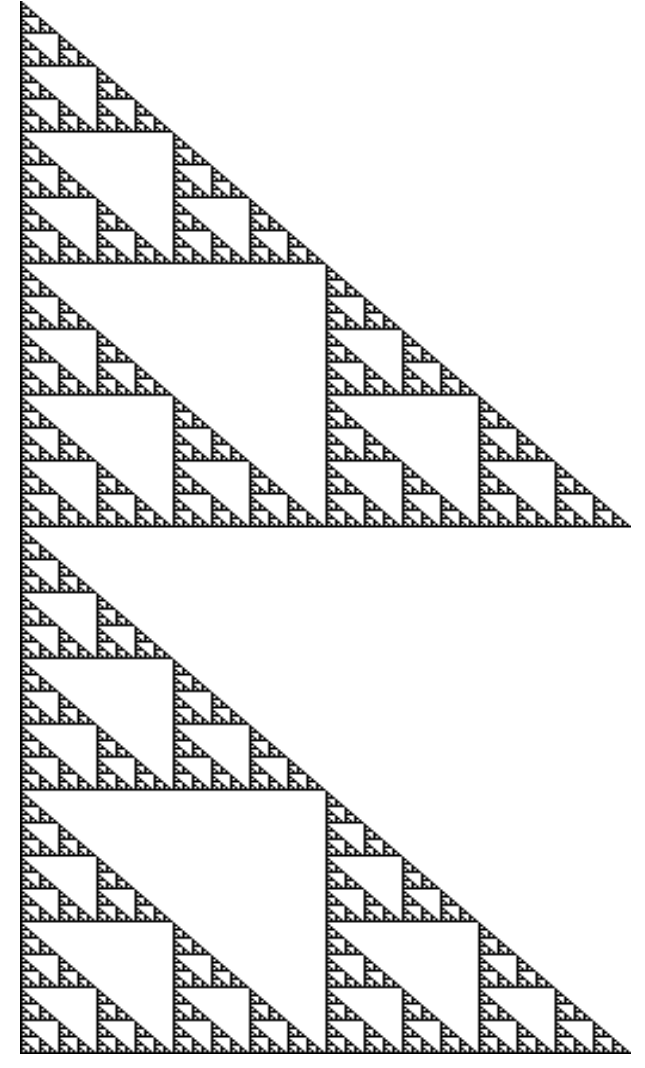

De-compressed Image (Adaptive Byte Fractal Decompression Output)

\section{Original image}

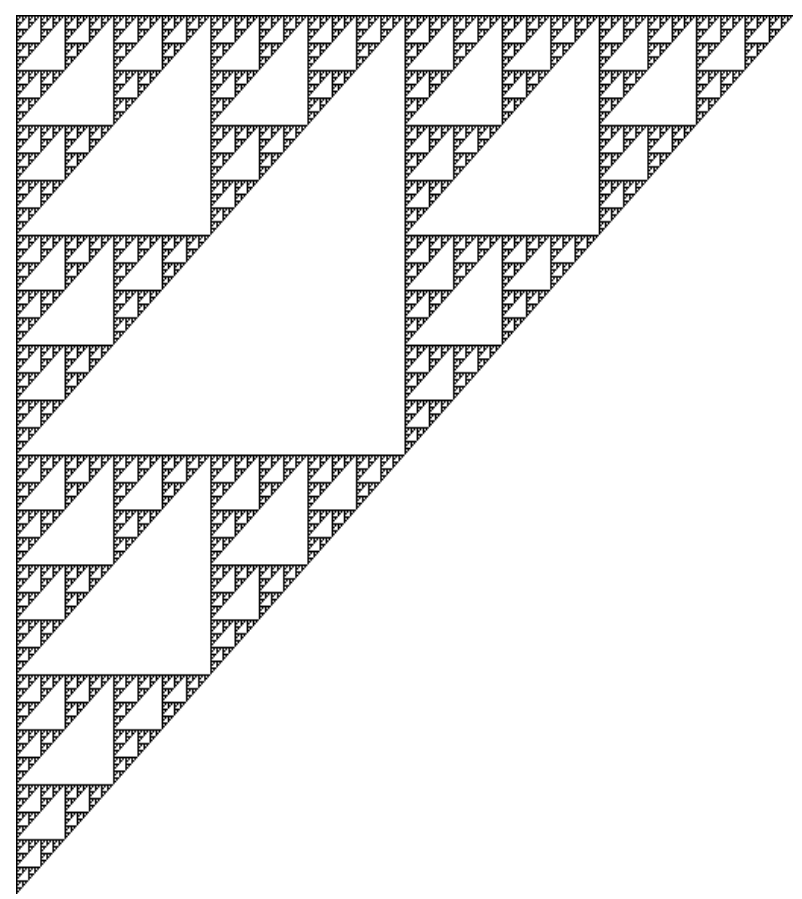

Figure 4. Signature4

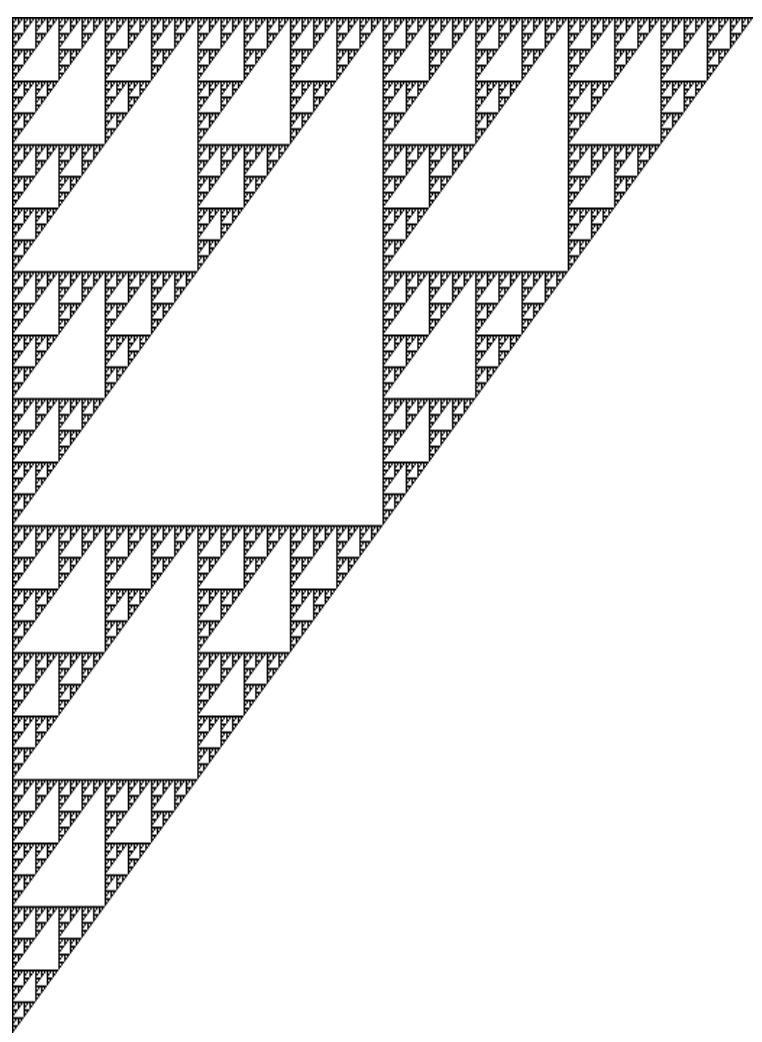

De-compressed Image (Adaptive Byte Fractal Decompression Output)

Original Image

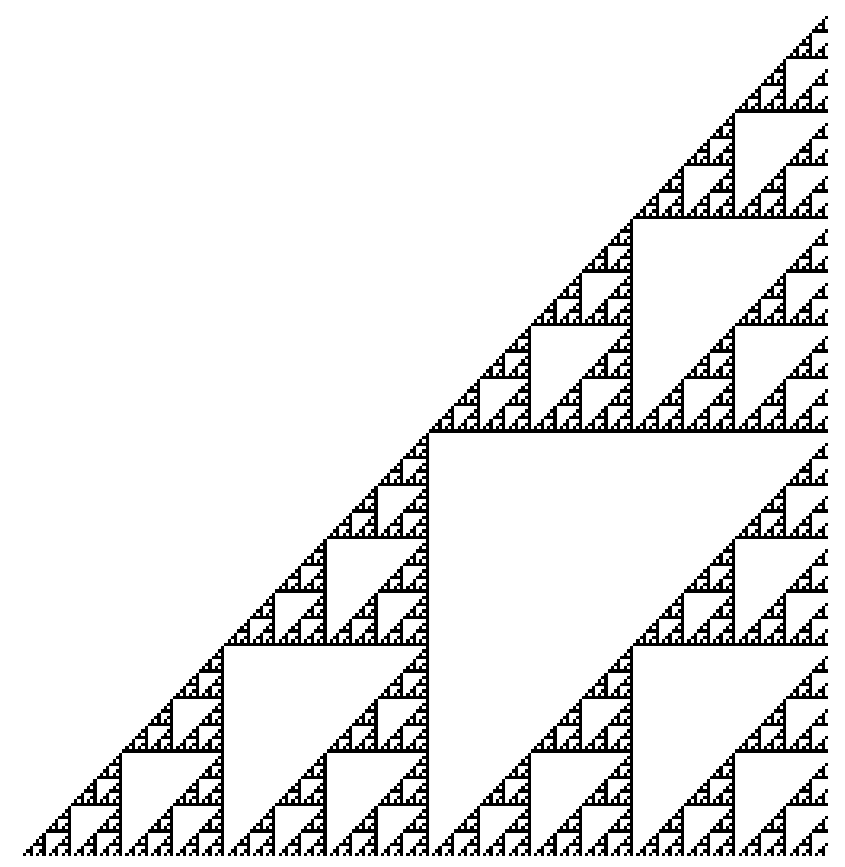

Figure 5. Signature5 


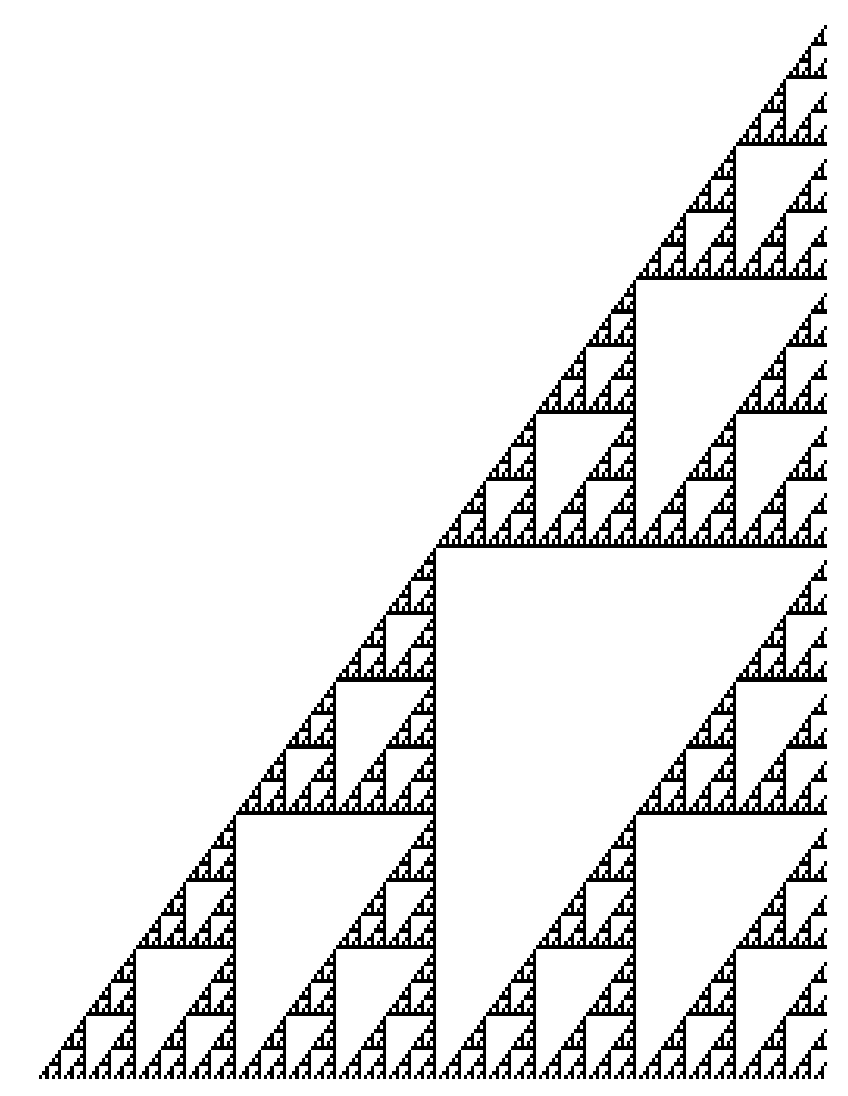

De-compressed Image (Adaptive Byte Fractal Decompression Output)

Original image

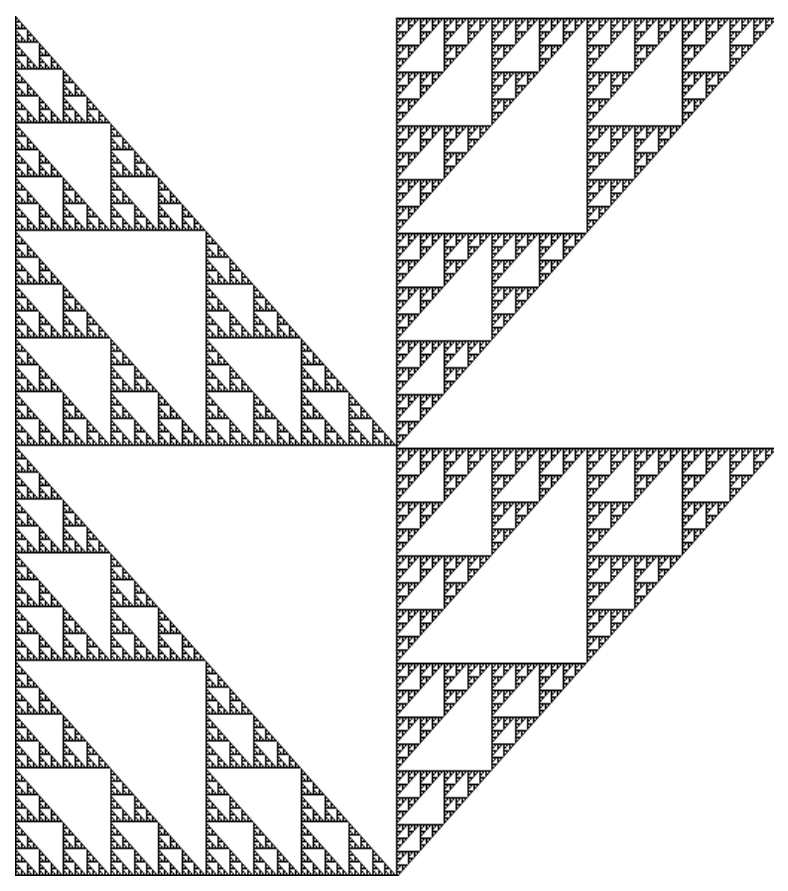

Figure 6. Signature6

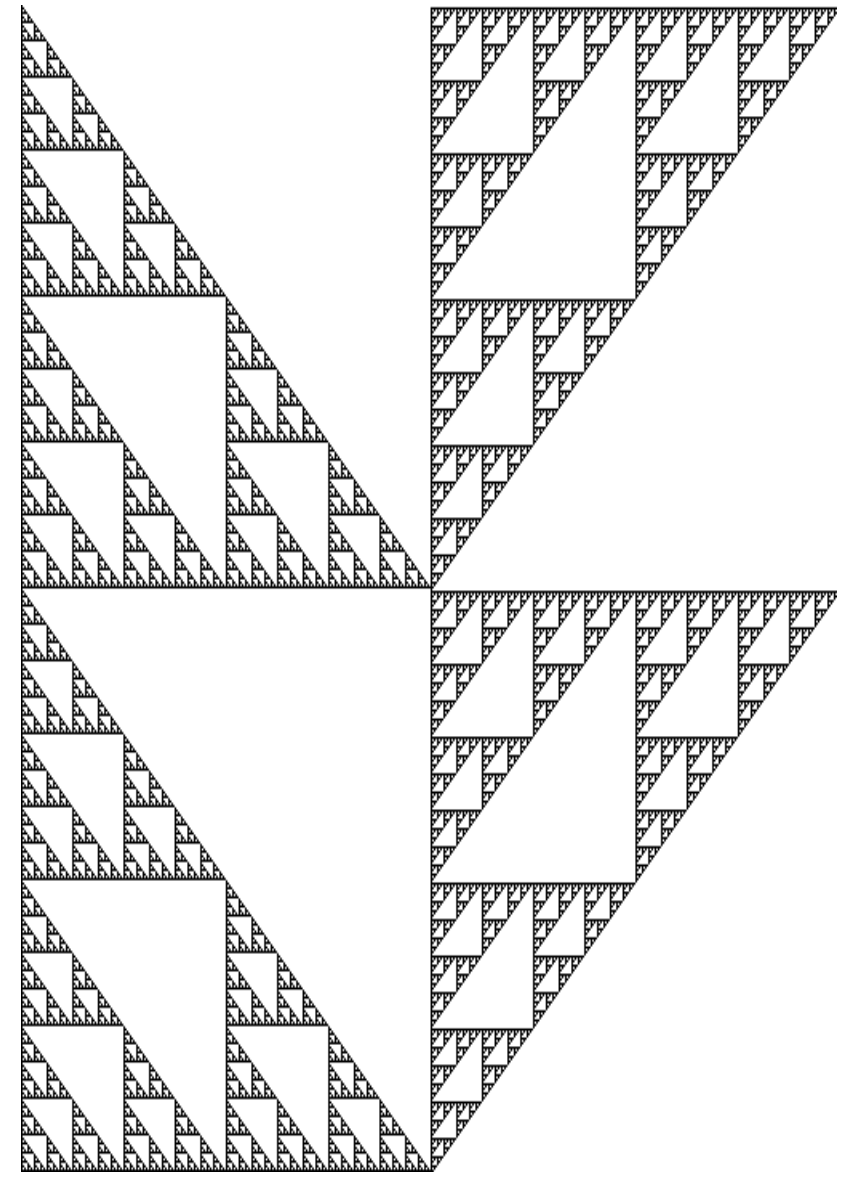

De-compressed Image (Adaptive Byte Fractal Decompression Output)

Original image

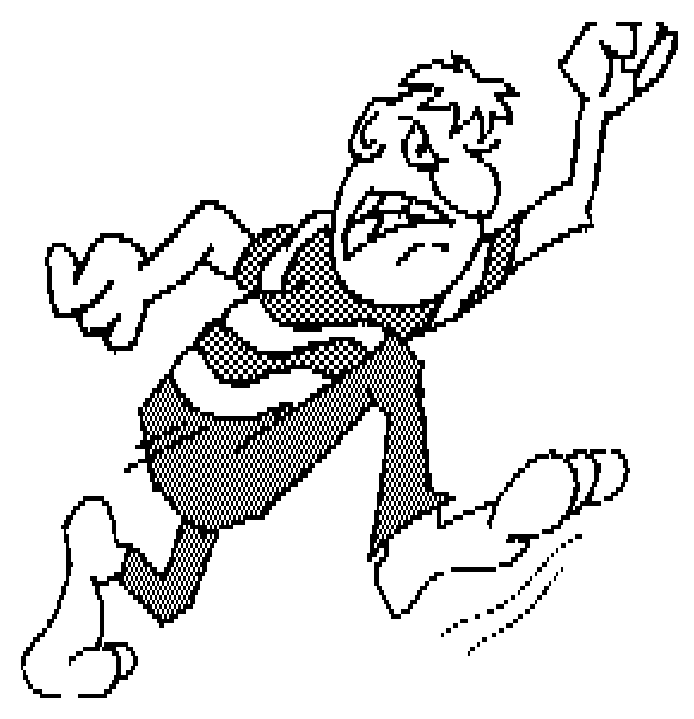

Figure 7. Portrait 


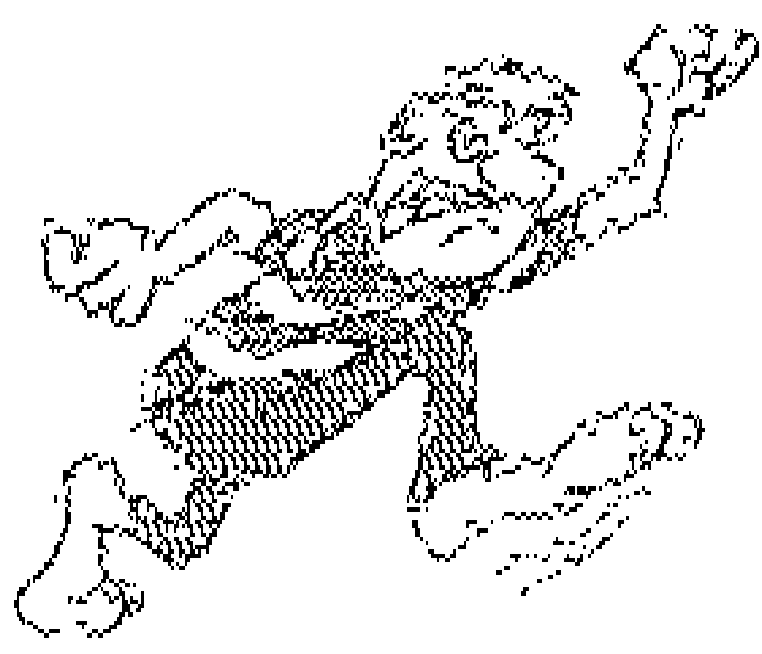

De-compressed Image (Adaptive Byte Fractal Decompression Output)

\section{Original image}

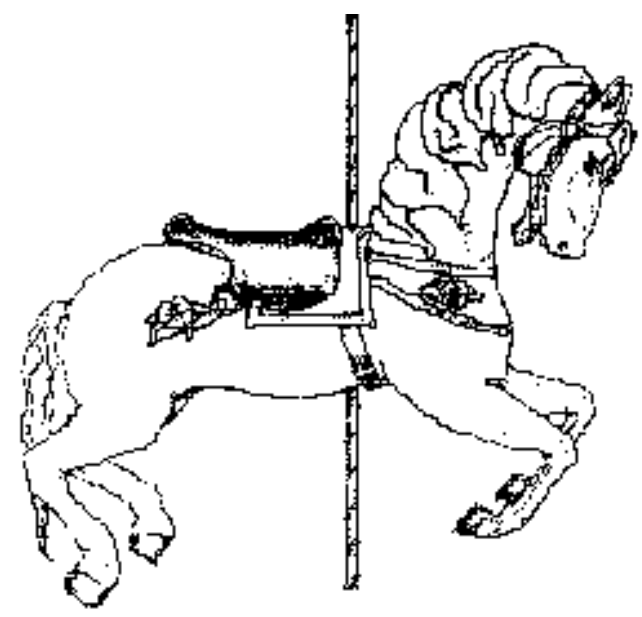

Figure 8. Horse

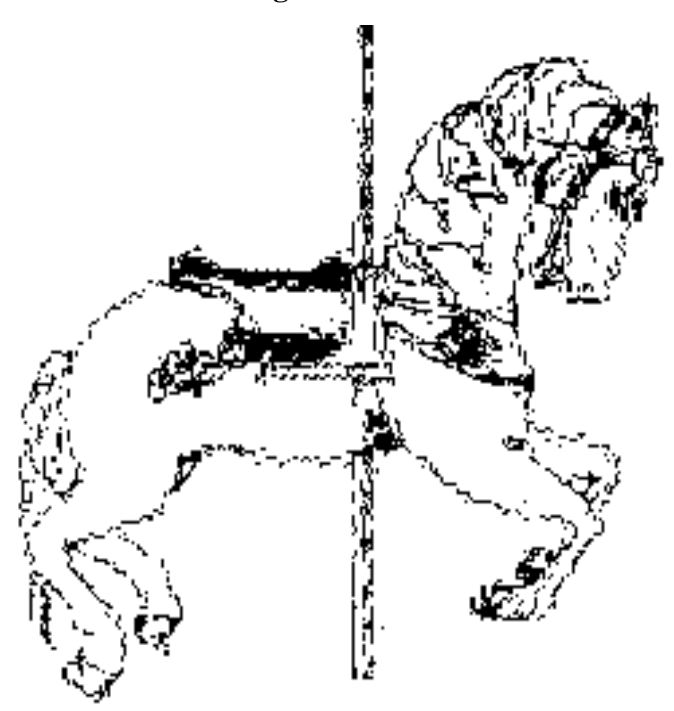

De-compressed Image (Adaptive Byte Fractal Decompression Output)
Original image

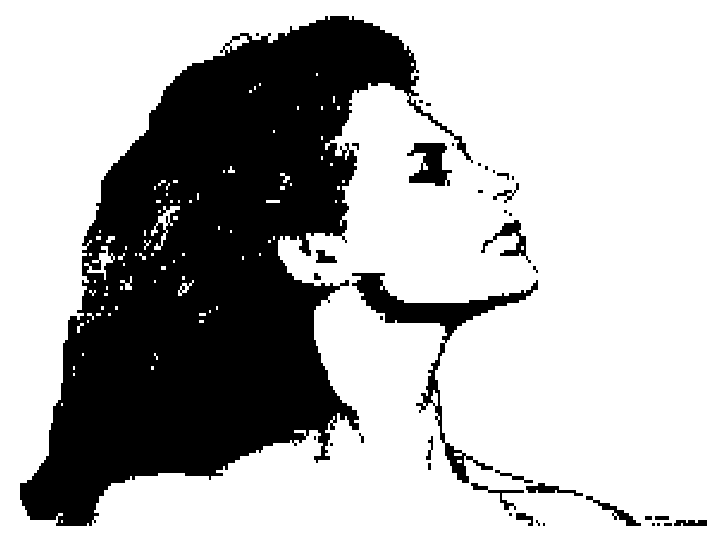

Figure 9. Breeze
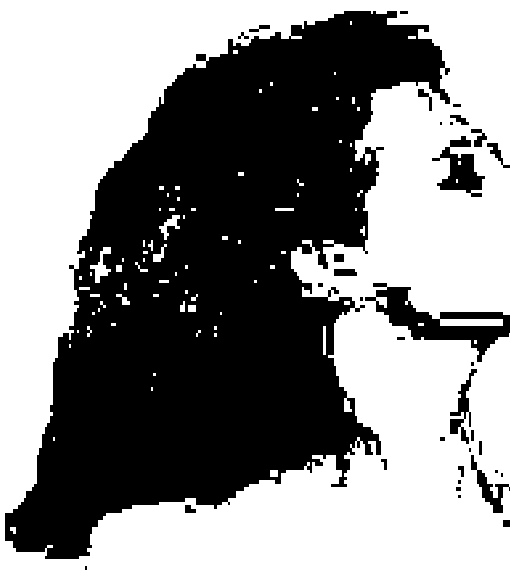

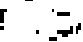

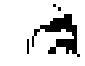

De-

compressed Image (Adaptive Byte Fractal De-compression Output)

\section{CONCLUSION}

Table 8.1. Comparison table in terms compression ratio

\begin{tabular}{|c|c|c|c|}
\hline SNo & Image & $\begin{array}{c}\text { Fractal } \\
\text { Compressi } \\
\text { on Ratio }\end{array}$ & $\begin{array}{c}\text { Adaptive } \\
\text { Byte Fractal } \\
\text { Compressio } \\
\text { n Ratio }\end{array}$ \\
\hline 1 & Signature1.bmp & $1.30: 1$ & $7.42: 1$ \\
\hline 2 & Signature2.bmp & $1.03: 1$ & $4.15: 1$ \\
\hline 3 & Signature3.bmp & $1.17: 1$ & $5.56: 1$ \\
\hline 4 & Signature4.bmp & $1.30: 1$ & $7.43: 1$ \\
\hline 5 & Signature5.bmp & $1.47: 1$ & $9.19: 1$ \\
\hline 6 & Signature6.bmp & $1.06: 1$ & $2.11: 1$ \\
\hline 7 & Portrait.bmp & $1.58: 1$ & $3.74: 1$ \\
\hline 8 & horse.bmp & $1.36: 1$ & $2.10: 1$ \\
\hline 9 & breeze.bmp & $1.45: 1$ & $3.16: 1$ \\
\hline
\end{tabular}


Table8.2. Comparison table in terms compression percentage

\begin{tabular}{|c|c|c|c|}
\hline S.No. & Image & $\begin{array}{l}\text { Fractal } \\
\text { compression } \\
\text { percentage }\end{array}$ & $\begin{array}{c}\text { Adaptive } \\
\text { Fractal } \\
\text { compression } \\
\text { percentage }\end{array}$ \\
\hline 1 & Signature1.bmp & 23.36 & 86.53 \\
\hline 2 & Signature2.bmp & 02.51 & 75.93 \\
\hline 3 & Signature3.bmp & 14.35 & 82.03 \\
\hline 4 & Signature4.bmp & 23.26 & 86.54 \\
\hline 5 & Signature5.bmp & 32.08 & 89.12 \\
\hline 6 & Signature6.bmp & 05.51 & 52.54 \\
\hline 7 & Portrait.bmp & 36.64 & 73.28 \\
\hline 8 & horse.bmp & 26.70 & 52.28 \\
\hline 9 & breeze.bmp & 31.05 & 68.37 \\
\hline
\end{tabular}

The table8.1. shows the comparison of fractal compression ratio and adaptive byte fractal compression ratio. As we can clearly see from table 8.1. Compression ratio is enhanced multiple times as evident from results, with limited compromise with the compression time .But this can be eliminated with multithreaded implementation of code and running on multi-tasking operating system, the compression time will not increase because in that case one thread will fill the fractal buffer and the second thread will do the adaptive compression.

The table8.2. indicates comparison in terms of compression percentage. It is clear from the table 8.2 that the percentage of adaptive fractal compression is much higher than the percentage of fractal compression.

Now little highlight on decompression of image-

- De-Compression time is minimally increased

- De-Compression results into original image or near to original image whose quality is same as fractal output.

\section{REFERENCES}

[1] Digital Image Processing by R. C. Gonzales and R. E. Woods, Addison-Wesley Publishing Company, 1992.

[2] Two-Dimensional Signal and Image Processing by J. S. Lim, Prentice Hall, 1990.

[3] Subramanya A, "Image Compression Technique," Potentials IEEE, Vol. 20, Issue 1, pp 19-23, Feb-March ,2001.

[4] David Jeff Jackson \& Sidney Joel Hannah, "Comparative Analysis of image CompressionTechniques," System Theory 1993, Proceedings SSST'93, 25th Southeastern Symposium,pp 513-517, 7 -9March 1993.

[5] Hong Zhang, Xiaofei Zhang \& Shun Cao, " Analysis \& Evaluation of Some Image Compression Techniques," High Performance Computing in AsiaPacific Region, 2000 Proceedings, 4th Int. Conference,vol. 2, pp 799-803,14-17 May, 2000

[6] Ming Yang \& Nikolaos Bourbakis ,"An Overview of Lossless Digital Image Compression Techniques," Circuits $\&$ Systems, 2005 48th Midwest Symposium ,vol. 2 IEEE ,pp 1099-1102,7 - 10 Aug, 2005.

[7] scz-compress.sourceforge.net

[8] [8].M. Nelson, The Data Compression Book, San Mako, CA: M \& T Publishing, Inc., 1992

[9] Lossless Data Compression, Report Concerning Space Data Systems Standards, CCSDS 120.0-G-2. Green Book. Issue 2. Washington, D.C.: CCSDS, December 2006.

[10] Gray, R. M. "Fundamentals of Data Compression",International Conference on Information, Communications, and Signal Processing Singapore, September 1997. IEEE Publication, New York.

[11] Sayood, Khalid, Lossless Compression Handbook, Elsevier Science, San Diego, CA, 2003.

[12] Barnsley, M.F, Hurd, L.P, "Fractal Image Compression", AK Peters, Wellesley, MA. (1993).

[13] Martin V Sewell, "Fractal Image Compression," MSc Computing Science project report, 1994.

[14] Bhumika Gupta, "Study Of Various Lossless Image Compression Technique", International Journal of Emerging Trends \& Technology in Computer Science (IJETTCS), Volume 2, Issue 4, July - August 2013. 